\title{
LA PROSPECCIÓN GEOELÉCTRICA Y ELECTROMAGNÉTICA EN DOS DIMENSIONES Y SU APLICACIÓN EN LA HIDROGEOLOGÍA
}

\author{
Mario Enrique Arias S. \\ Escuela Centroamericana de Geología, Apdo. 2-14, 2060 \\ Universidad de Costa Rica, San José \\ E-Mail: marioa@geologia.ucr.ac.cr
}

\begin{abstract}
The benefits of the geophysical prospection are shown in its two dimensions, because it allows to obtain a more precise vision of the lateral and vertical variations. For this reason the application of the electric and electromagnetic methods is exposed together with two examples done in coastal aquifers of Costa Rica.
\end{abstract}

RESUMEN: Se presentan los beneficios de la prospección geofísica en dos dimensiones, pues permite obtener una visión más clara de las variaciones laterales y a profundidad. Para ello se ha introducido la aplicación de los métodos eléctricos y electromagnéticos con dos ejemplos realizados en acuíferos costeros de Costa Rica.

\section{ANTECEDENTES}

Los métodos geoeléctricos han sido y son utilizados como práctica común en la investigación hidrogeológica para obtener información de las propiedades del subsuelo. Se han empleado métodos que operan por corriente continua y utilizando un dispositivo de cuatro electrodos que se sitúan sobre el terreno en contacto eléctrico con el medio rocoso a estudiar.

Respecto al arreglo de electrodos existe una notable variedad de dispositivos de medida, lo cual confiere a este tipo de medidas una gran flexibilidad para investigar variaciones en la distribución de la resistividad del subsuelo. Tradicionalmente en nuestro país, la aplicación de la prospección geofísica con fines hidrogeológicos se ha enfocado al uso de los métodos eléctricos en una dimensión (1D), principalmente sondeos eléctricos verticales (SEV) con dispositivos del tipo Schlumberger y Wenner, ejemplos de ello son los trabajos desarrollados por Arias (1996, 2000a).

En los últimos años, el surgimiento de nuevas técnicas geofísicas, especialmente en dos dimensiones (2D), están ayudando a resolver problemas que se presentan con gran frecuencia al hidrogeólogo, como son la caracterización de la contaminación, su evolución temporal y espacial y origen del problema del deterioro de las aguas subterráneas.

\section{LA TÉCNICA DE TOMOGRAFÍA ELÉCTRICA}

El desarrollo de la tomografía eléctrica y su efectiva inversión e interpretación con la ayuda de programas de computo hace de esta técnica una herramienta muy útil en las prospecciones 
superficiales con fines de evaluación espacial y temporal del flujo de agua subterránea. La tomografía eléctrica pretende obtener una imagen de las propiedades eléctricas del subsuelo y para ello se utilizan una serie de electrodos hincados en el suelo a un intervalo igual de separación a lo largo de una línea de perfil. En las prospecciones superficiales los electrodos se encuentran separados a una distancia entre 1 y $15 \mathrm{~m}$ en función de la profundidad de investigación deseada. Un cable multicanal conecta cada electrodo al sistema central que mide y almacena la resistividad del subsuelo.

La información es procesada e interpretada con la ayuda de programas de cómputo que permiten una inversión correcta de los datos, evitando efectos de borde. Sin embargo, debido al alto costo económico de los programas muchas veces solamente se obtiene una imagen formada por curvas de igual resistividad, denominada pseudosección eléctrica. En esta metodología, se dibujan los datos de resistividad en función de la profundidad de la sección; los datos de resistividad aparente son considerados como el valor de resistividad real en el punto al aplomo del centro del dispositivo de medición, a una profundidad proporcional a la separación entre los electrodos.

Para obtener la resistividad del subsuelo, se inyecta una corriente por un par de electrodos (A y B) y se mide el voltaje generado en otros dos electrodos ( $\mathrm{M}$ y N). Para construir una imagen que muestre las variaciones de la resistividad del subsuelo a lo largo de un sector, es necesario realizar un perfil de mediciones en las cuales la separación de los electrodos esté definida por una distancia $a$, el espacio entre los electrodos debe incrementarse en $2 a$ para el nuevo perfil. El proceso se repite incrementando la separación de los electrodos cada vez en múltiplos $N$ de la separación $a$ (Fig. 1). Conforme la separación de los electrodos es mayor; la profundidad de investigación se incrementa (Griffith \& Barker, 1994).

Con la utilización de un dispositivo Wenner, Polo-Polo o Dipolo-Dipolo, el valor de la resistividad eléctrica corresponde con la parte central de los cuatro electrodos, y su profundidad es usualmente $a / 2$. Los datos pueden ser interpolados y unidos por medio de líneas de isoresistivi-

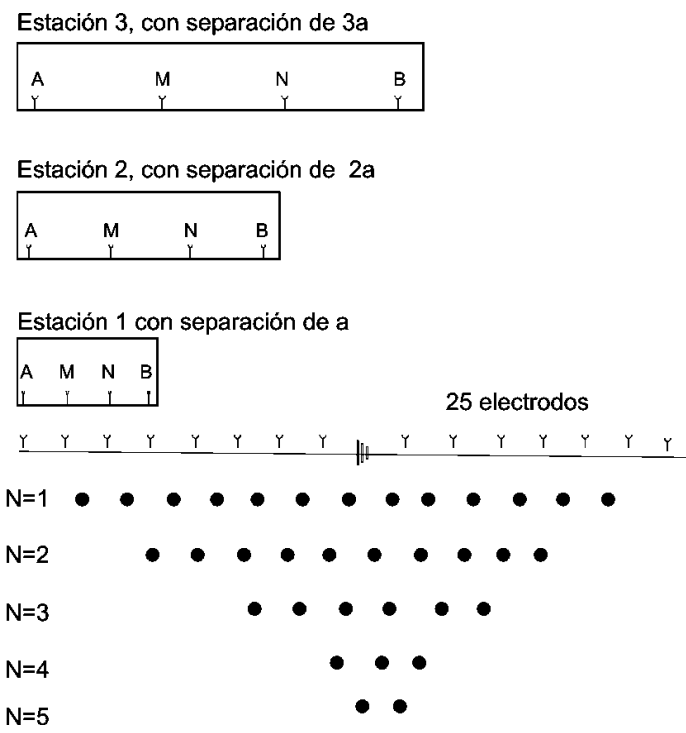

Fig.1: Secuencia de medición de la resistividad del suelo para la construcción de una pseudosección eléctrica.

dad obteniendo una pseudosección en 2D, que refleja la distribución de la resistividad en función de la profundidad y de la distancia horizontal.

Barker (1992) ha desarrollado un algoritmo para la inversión de los datos de tomografía a partir de la pseudosección eléctrica; para ello se utiliza el valor de la resistividad aparente como el modelo inicial para la integración con modelo finito de dos dimensiones. Las diferencias observadas entre las imágenes procesadas por el computador y la medida en el campo son usadas para aplicar correcciones al modelo.

La técnica de inversión automatizada, generalmente está basada en el método de suavizado de mínimos cuadrados, que produce un modelo de dos dimensiones a partir de los valores de resistividad aparente de la pseudosección. Este modelo presentará la profundidad y resistividad real del subsuelo. En la actualidad, los dos programas más utilizados para realizar esta inversión y convertir la pseudosección en una imagen de tomografía eléctrica son el RESIXIP2D o el RES2DINV.

La pseudosección es una aproximación del subsuelo, ya que cada valor es afectado por la resistividad aparente que incluye una porción de suelo sobre y debajo del punto de medición. Por 
lo tanto refleja la geología superficial pero debido a la geometría de los electrodos presenta cierto grado de distorsión; tanto es así que investigaciones realizadas con diferentes dispositivos eléctricos sobre el mismo sitio, pueden brindar resultados diferentes. Esta es una limitación importante en este tipo de representaciones que se puede corregir con una inversión apropiada de los datos (Olmo \& López, 1999). Diferentes ejemplos de aplicación se han realizado en nuestro país, varios de ellos desarrollados por Arias (2000b, 2000c y 2002a).

\section{PERFILAJE ELECTROMAGNÉTICO}

Los métodos electromagnéticos (EM), tal como los eléctricos, permiten una mejor comprensión del subsuelo gracias al estudio de la resistividad o, de su valor inverso, la conductividad de las formaciones que lo componen. En el caso de los métodos electromagnéticos la corriente es oscilante y por lo tanto asociada a ondas electromagnéticas y en este caso específico es por inducción y no por medio de electrodos que se inyecta al suelo.

Para que este método sea eficiente, tres premisas se deben cumplir en toda prospección (Meyer de Stadelhofen, 1995):

a) Algunas formaciones del subsuelo deben presentar características conductoras para que las corrientes eléctricas inducidas puedan ser detectadas.

El campo magnético primario proveniente del emisor debe penetrar suficiente en el subsuelo.
El campo secundario desarrollado en las formaciones conductoras del subsuelo debe ser percibido en superficie.

La instrumentación del tipo EM-34 mide la conductividad del suelo en miliSiemens/metro. Sus principales componentes son dos bobinas las cuales son conectadas por medio de un cable de referencia al transmisor y al receptor de la señal electromagnética. La separación de las bobinas determina la resolución lateral y la profundidad de penetración. Según el fabricante (Geonics, 1980) este instrumento está diseñado para operar bajo las siguientes características que se muestran en el cuadro 1.

La metodología de trabajo en el campo consiste en la realización de perfiles a diferente profundidad, en función de la separación de las bobinas y de la posición de las mismas. Así es posible obtener hasta seis datos en cada punto de medición de un perfíl, lo cual permite realizar interpretaciones en función de la profundidad y de las variaciones laterales. Para la realización de un perfil se requiere de dos personas y en condiciones ideales se podría prospectar hasta $5 \mathrm{~km}$ diarios.

En la literatura, se reportan gran cantidad de aplicaciones, entre las que destacan (Geonics, 1992): determinación de zonas fracturadas que favorecen la porosidad secundaria en acuíferos; cartografiado de zonas altamente conductoras dentro del mismo acuífero, que en la mayoría de los casos son relacionadas con plumas de contaminación; determinación de la extensión de la intrusión salina en acuíferos costeros; identificación de cavidades en rocas carbonatadas y caracterización de tipos de suelo, entre otros.

Cuadro 1

Características de medición del EM-34

\begin{tabular}{cccc}
\hline Separación entre bobinas $(\mathrm{m})$ & $\begin{array}{l}\text { Frecuencia de } \\
\text { medición }(\mathrm{Hz})\end{array}$ & Profundidad de exploración $(\mathrm{m})$ \\
\hline & & Dipolo horizontal & Dipolo Vertical \\
\hline 10 & 6400 & 7,5 & 15 \\
20 & 1600 & 15 & 30 \\
40 & 400 & 30 & 60 \\
\hline
\end{tabular}




\section{EJEMPLOS DE APLICACIÓN}

A continuación se presentan los resultados parciales de dos estudios realizados en acuíferos costeros de Costa Rica aplicando la pseudosección eléctrica y el perfilaje EM-34.

\section{Acuífero de Sámara}

Arias (2002a) realizó una pseudosección (Fig.2) en las proximidades del pozo que abastece de agua al acueducto de Sámara. En ella se puede observar, a partir de los 25 metros de iniciada, la existencia de cuerpos conductores de morfología un poco irregular, lo cual hace pensar en cuerpos lenticulares vistos desde una perspectiva transversal, que tienden a ser más prominentes al final de esta imagen.

En la figura 2 la zona saturada está representada por valores de resistividad que varían entre los 250 y 450 Ohm*m; su morfología es muy singular y hace pensar en al menos tres interpretaciones. La primera de ellas corresponde con la existencia de paleocanales, pues la morfología es típica de estas estructuras sedimentarias. La segunda interpretación tiene que ver con un control estructural, el cual estaría delimitando las características acuíferas del material aluvional existente. La última es una conjunción de las dos anteriores, en la cual la instalación de los paleoca- nales está controlada por la existencia de grábenes y horsts. Posiblemente esta interpretación es la que mejor explique la génesis del acuífero aluvial de Sámara, pues algo similar existe en otras secciones descritas.

\section{Acuífero de Tamarindo}

Como parte del estudio en el acuífero de Tamarindo, la prospección geofísica realizada por Arias (2002b) incluyó entre otras cosas un perfil electromagnético de 700 metros de longitud a lo largo de la pista del aeropuerto de la localidad (Fig.3). La investigación electromagnética incluyó la elaboración de perfiles utilizando tanto el dipolo vertical como el horizontal, con separación entre las bobinas de 10, 20 y 40 m. En esta prospección se aprecia como el valor de la conductividad eléctrica varía lateralmente y en la profundidad, brindando información sobre las variaciones litológicas de la zona.

La morfología de los perfiles es muy similar entre ellos; mostrando la existencia de sectores conductores con valores del orden de hasta 90 $\mathrm{mS} / \mathrm{m}$, equivalente a $11 \mathrm{Ohm} * \mathrm{~m}$ y sectores resistivos con valores de $5 \mathrm{mS} / \mathrm{m}$ (200 Ohm*m). La interpretación está relacionada con los depósitos aluvionales del río San Andrés, el cual en este sector se caracteriza por la existencia de gran cantidad de meandros de reducido tamaño, lo

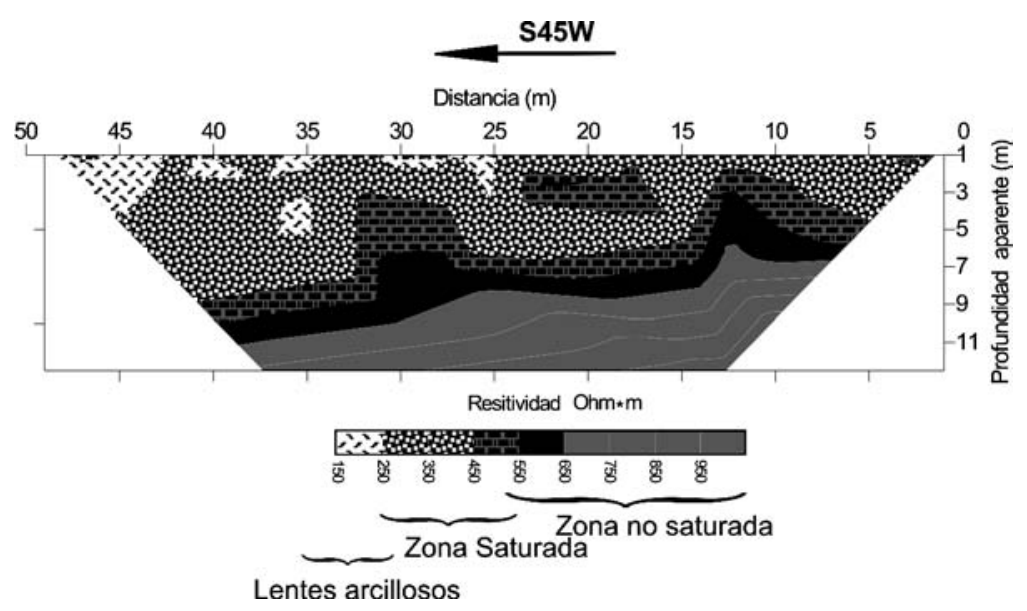

Fig. 2: Pseudosección eléctrica utilizando un dispositivo Polo - Polo, ubicada en el pozo del acueducto de Sámara. 

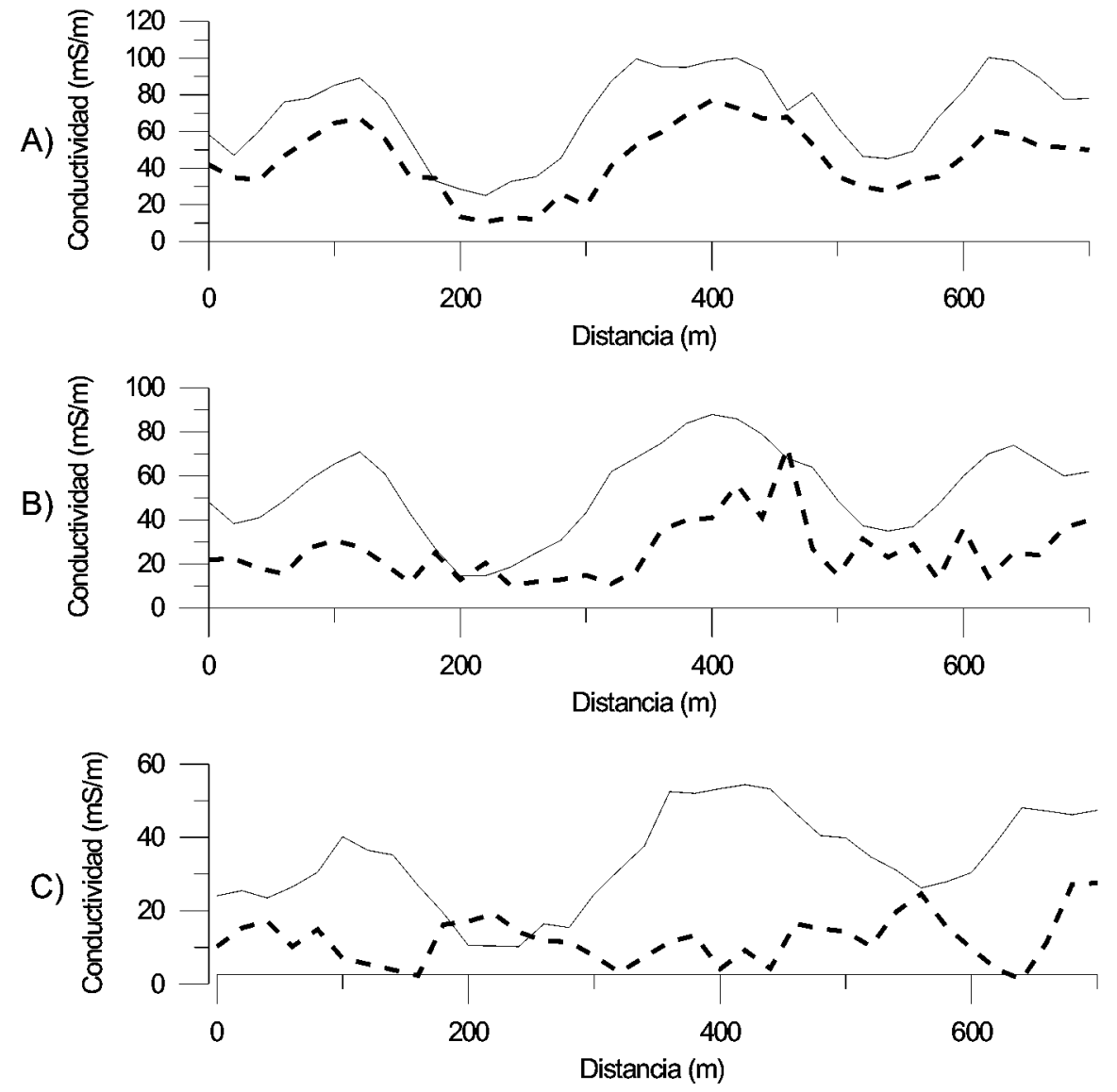

Fig. 3: Perfiles electromagnéticos realizados en la pista del aeropuerto de Tamarindo.

A) Separación entre bobinas de $10 \mathrm{~m}$. B) Separación entre bobinas de $20 \mathrm{~m}$. C) Separación entre bobinas de 40 m. Línea contínua representa el dipolo horizontal y línea discontínua corresponde con el dipolo vertical.

cual hace pensar en procesos de depositación de materiales finos como limos y arcillas que caracterizan los sectores conductores y, por otra parte, la depositación de arenas y gravas que corresponden con los sectores resistivos.

La aplicación directa de esta prospección ha delimitado los sectores más apropiados para la instalación de nuevos pozos y ha brindado indicios para establecer un modelo conceptual del acuífero, pues la alternancia lateral de materiales conductores y resistivos limita y condiciona la productividad del mismo; por lo tanto, es una herramienta que orienta al hidrogeólogo en la interpretación y toma de decisiones.

\section{CONSIDERACIÓN FINAL}

La aplicación de nuevas técnicas de medición e interpretación de datos geofísicos, en este caso utilizando una metodología en la cual se representan las variaciones laterales y a profundidad, es sin duda una herramienta de gran valor para el hidrogeólogo, pues permite obtener una visión bidimensional de las características litológicas asociadas al acuífero.

Sin embargo, para que estas metodologías sean de uso frecuente en diferentes ramas de las geociencias, es necesario promover la adquisición de tecnología avanzada y programas 
de interpretación con el fin de evitar los problemas de equivalencia; además, de potencializar la eficacia tanto en tiempo como en calidad de los datos adquiridos en este tipo de prospección.

\section{REFERENCIAS}

ARIAS, M.E., 1996: Evaluación del riesgo de contaminación del acuífero de Jacó, cantón de Garabito, Puntarenas. - 48 págs, SENARA, San José [Inf. interno].

ARIAS, M.E., 2000a: Informe de prospección geoléctrica en Home Creek, Cahuita, Limón. - 10 págs. Sec. Sismol. Vulcanol. Explor. Geofísica, Univ. de Costa Rica, San José [Inf. interno].

ARIAS, M.E., 2000b: Informe de prospección geoléctrica en la Isla del Coco, Costa Rica. - 12 págs. Sec. Sismol. Vulcanol. Explor. Geofísica, Univ. de Costa Rica, San José [Inf. interno].

ARIAS, M.E., 2000c: Prospección geofísica para aguas subterráneas. - Escuela Centroamericana de Geología, UCR, San José [Informe de gira curso SP1133].

ARIAS, M.E. 2002a: Evaluación geofísica en Sámara, Costa Rica. Proyecto Evaluación del Acueducto de Sáma- ra. - 9 págs. SENARA - Univ. de Costa Rica, San José [Inf. interno].

ARIAS, M.E. 2002b: Evaluación geofísica en Tamarindo; Costa Rica. - 8 págs. SENARA - Univ. de Costa Rica, San José [Inf. interno].

BARKER, R. 1992: A simple algorithm for electrical imaging of the subsurface. - First Break, 10: 53-62.

GEONICS, 1980: Electromagnetic terrain conductivity measurement at low induction numbers. - 15 págs. Ontario [Technical Note].

GEONICS, 1992: Groundwater exploration applications. - 23 págs. Ontario [Technical Note].

GRIFFIHTS, D. H. \& BARKER, R. D. 1994: Electrical imagin in archaelogy. - J. Archaelogical Sci. 21: 153158.

MEYER DE STADELHOFEN, C. 1995: Applications de la géophysique aux recherches d'eau. - 177 págs. Lavoisier, Paris.

OLMO, M. \& LÓPEZ, J., (eds.) 1999: Actualidad de los técnicas geofísicas. - 375 págs. Inst. Tecnol. Geominero de España, Madrid. 